\title{
Erratum: Relativistic parameterizations of neutron matter and implications for neutron stars [Phys. Rev. C 98, 065804 (2018)]
}

\author{
Nadine Hornick, Laura Tolos, Andreas Zacchi $\odot$, Jan-Erik Christian, and Jürgen Schaffner-Bielich
}

(Received 23 November 2020; published 23 March 2021)

DOI: 10.1103/PhysRevC.103.039902

We discovered an error in our code for calculating the tidal deformability. The other results of the paper are unaffected by this error. Only Table I needs to be corrected for the numerical values of the tidal deformability. It now reads:

TABLE I. Central energy density, central pressure, radius, and dimensionless tidal deformability of a $1.4 M_{\odot}$ neutron star for different values of $m^{*} / m$ and for a slope parameter of $L=60 \mathrm{MeV}$ and a symmetry energy of $J=32 \mathrm{MeV}$.

\begin{tabular}{|c|c|c|c|c|c|c|c|}
\hline$m^{*} / m$ & $\epsilon_{c}\left(1.4 M_{\odot}\right)\left[\frac{\mathrm{MeV}}{\mathrm{fm}^{3}}\right]$ & $p_{c}\left(1.4 M_{\odot}\right)\left[\frac{\mathrm{MeV}}{\mathrm{fm}^{3}}\right]$ & $R_{1.4 M_{\odot}}(\mathrm{km})$ & $\Lambda_{1.4 M_{\odot}}$ & $\epsilon_{c}\left(2 M_{\odot}\right)\left[\frac{\mathrm{MeV}}{\mathrm{fm}^{3}}\right]$ & $p_{c}\left(2 M_{\odot}\right)\left[\frac{\mathrm{MeV}}{\mathrm{fm}^{3}}\right]$ & $M_{\max }\left(M_{\odot}\right)$ \\
\hline 0.55 & 268 & 32.2 & 13.9 & 960 & 329 & 64.2 & 2.90 \\
\hline 0.60 & 302 & 37.0 & 13.5 & 811 & 381 & 79.0 & 2.72 \\
\hline 0.70 & 397 & 53.4 & 12.8 & 535 & 590 & 150 & 2.32 \\
\hline 0.75 & 466 & 65.7 & 12.4 & 422 & 856 & 261 & 2.11 \\
\hline
\end{tabular}

This changes our conclusion slightly. Now the cases of $m^{*} / m \geqslant 0.65$ are within the $90 \%$ confidence level of the limit of $\Lambda<720$ from GW170817 [1] instead of $m^{*} / m>0.65$.

We took this opportunity to change the crust equation of state (EoS) to the Baym, Pethick, and Sutherland [2] and Negele and Vautherin [3] equation of state in order to improve matching. However, we note that the effects of a crust EoS on the tidal deformability are known to be less than $11 \%$ [4].

We thank D. Chatterjee for pointing out the error in our code.

[1] B. P. Abbott et al. (LIGO Scientific Collaboration and Virgo Collaboration), Phys. Rev. Lett. 119, 161101 (2017).

[2] G. Baym, C. Pethick, and P. Sutherland, Astrophys. J. 170, 299 (1971).

[3] J. W. Negele and D. Vautherin, Nucl. Phys. A 207, 298 (1973).

[4] A. M. Kalaitzis, T. F. Motta, and A. W. Thomas, Int. J. Mod. Phys. E 28, 1950081 (2019). 